\title{
Transient and steady-state response properties of movement detectors
}

\author{
Martin Egelhaaf and Alexander Borst \\ Max-Planck-Institut für Biologische Kybernetik, Spemannstrasse 38, D-7400 Tübingen, \\ Federal Republic of Germany
}

Received January 4, 1988; accepted August 25, 1988

\begin{abstract}
The transient and steady-state responses of movement detectors are studied at various pattern contrasts (i) by intracellularly recording from an identified movement-sensitive interneuron in the fly's brain and (ii) by comparing these results with computer simulations of an array of movement detectors of the correlation type. At the onset of stimulus motion, the membrane potential oscillates with a frequency corresponding to the temporal frequency of the stimulus pattern before it settles at its steady-state level. Both the transient and the steady-state response amplitudes show a characteristic contrast dependence. As is shown by computer modeling, the transient behavior that we found in the experiments reflects an intrinsic property of the general scheme of movement detectors of the correlation type. To account for the contrast dependence, however, this general scheme has to be elaborated by (i) a subtraction stage, which eliminates the background light intensity from the detector input signal, and (ii) saturation characteristics in both branches of each movement-detector subunit.
\end{abstract}

\section{INTRODUCTION}

The extraction of motion information from the changing light-intensity distribution across the photoreceptors is a basic problem for the visual system. The principal mechanisms of motion detection have been studied intensively in various species and at different levels of analysis, comprising behavioral, psychophysical, neurophysiological, and theoretical approaches. ${ }^{1-5}$ Of the different models of biological motion detection, the movement detector of the so-called correlation type is perhaps the best established one. Originally it was proposed to account for motion perception in insects, ${ }^{1,3,6-9}$ but recently it was shown also to account for various aspects of motion vision in humans. ${ }^{10-14}$ In brief, the mechanism of motion detection is nonlinear and local. It is based on the multiplicationlike interaction of the appropriately filtered signals of neighboring retinal input channels.

In most theoretical accounts of this motion-detection scheme, as well as its alternative formulations, the steadystate responses of the detector were mainly taken into account. ${ }^{1,12,15-17}$ There are only a few studies that concentrate on the dynamical aspects of the motion-detector theory. ${ }^{18-20}$ Particularly important in this context was the finding that the steady-state responses of movement detectors cannot account for recent experimental data obtained under transient stimulus conditions ${ }^{20,21}$ and might even result in misleading interpretations of experimental results. This prompted us to examine, both theoretically and experimentally, the transient responses of movement detectors of the correlation type to the onset of pattern motion. The transient characteristics are analyzed as a function of stimulus velocity and pattern contrast and are compared with their steady-state counterparts. The specific relationship between pattern contrast and the response amplitudes are then used to elaborate the motion-detection scheme in a simple and physiologically plausible way.
As our experimental paradigm, we used the visual system of the fly because it has long been established as a good model system for studying various motion-dependent visual information-processing tasks. ${ }^{22}$ We recorded from a motion-sensitive interneuron, the horizontal (HS) cell, in the fly's brain, which proved to be advantageous from an analytical point of view. First, this cell can be identified individually from preparation to preparation. ${ }^{23}$ Second, it can be recorded intracellularly, which allows us to monitor the summed synaptic potentials of its presynaptic elements exactly. Third, because these presynaptic elements are assumed to represent the local movement detectors, biological motion detection can conveniently be analyzed without much interference from other processes. The importance of the last point cannot be overestimated if we are interested mainly in the basic mechanisms underlying motion detection. Additional processing might complicate the interpretations when we try to analyze the mechanisms of motion detection on the basis of behavioral or psychophysical experiments. However, only if the properties of the movement detectors are known is it possible to understand the role of the subsequent information-processing steps, which are essential for doing well in the visual world.

\section{MATERIAL AND METHODS}

\section{A. Electrophysiology}

1. Preparation

Female blowflies (Calliphora erythrocephala) were briefly anesthesized with $\mathrm{CO}_{2}$ and mounted ventral side up with wax on a small preparation platform. The head capsula was opened from behind, and the trachea and air sacs, which normally cover the lobula plate, were removed. To eliminate movements of the brain caused by peristaltic contractions of the esophagus, the proboscis of the animal was cut away, and the gut was pulled out. This allowed us to make 
stable intracellular recordings of up to $45 \mathrm{~min}$. During the experiment, the fly was supplied with ringer solution through the indifferent electrode. ${ }^{24}$

\section{Recording}

Electrodes were pulled on a Brown-Flaming micropipette puller (P-77), using glass capillaries with a diameter of $1 \mathrm{~mm}$ (Clark, GC100F-10). They were filled with $1 \mathrm{M} \mathrm{KCl}$ and had resistances of approximately 30-50 M $\Omega$. For further data analysis the graded potentials of the HS cells recorded in response to movement stimuli were fed to an IBM AT computer through a 12-bit analog-to-digital converter (Data Translation Model DT2801-A) at a sampling rate of $2 \mathrm{kHz}$. The programs for the evaluation of the data were written in ASYST (Macmillan Software Company, Keithley Instruments).

\section{Stimulation}

A monitor (Tectronix 608) was placed in front of the right eye at an angle of $45^{\circ}$ from the fly's frontal midline. The position of the fly was carefully adjusted by using the symmetry of the frontal equatorial pseudopupils of both eyes. ${ }^{25}$ As seen by the fly, the display had a horizontal angular extent of $68^{\circ}$ and a vertical extent of $81^{\circ}$. The stimulus pattern was produced by an image synthesizer (Picasso, Innisfree Inc.) controlled by an IBM AT. The intensity of the pattern was modulated sinusoidally along its horizontal axis. The stimulus grating had a fixed wavelength of $17^{\circ}$; its contrast could be varied between 0.05 and 0.5 . The mean luminance of the pattern was approximately $25 \mathrm{~cd} / \mathrm{m}^{2}$. In the experiments shown here the pattern was moved from back to front for $3.6 \mathrm{sec}$; between the different presentations of stimulus motion, the pattern was kept stationary for 4.7 sec. The pattern velocity differed in different experiments, as will be indicated in Section 3 .

\section{B. Computer Simulations}

A one-dimensional array of as many as 72 correlation-type movement detectors (Fig. 2) per spatial period was simulated on an IBM AT by using the ASYST software. The relevant details of the computer simulations will be given in Section 3.

\section{RESULTS}

As an indicator for the performance of the fly's motiondetection system, we used the visually induced response of the so-called HS cells, which reside in the posterior part of the fly's third visual ganglion, the lobula plate. Until this stage is reached, the retinotopic order of the visual pathway remains preserved because of a columnar organization of the visual ganglia. This is schematically shown in Fig. 1 . In the lobula plate the point-to-point representation of visual space is abandoned by a set of large-field integrating visual interneurons. ${ }^{23}$ Because these cells show an extraordinary structural constancy and highly invariant physiological characteristics, they can be identified individually from preparation to preparation. The three HS cells on each side of the fly's brain belong to this group of neurons. Jointly they cover the entire extent of the ipsilateral visual hemisphere with their receptive fields. In the lobula plate they receive input from a large number of retinotopically organized columnar elements, which are assumed to represent local movement detectors. ${ }^{24,26}$ The HS cells are sensitive to movement along the horizontal axis of the eye in a directionally selective way: when stimulated with periodic patterns

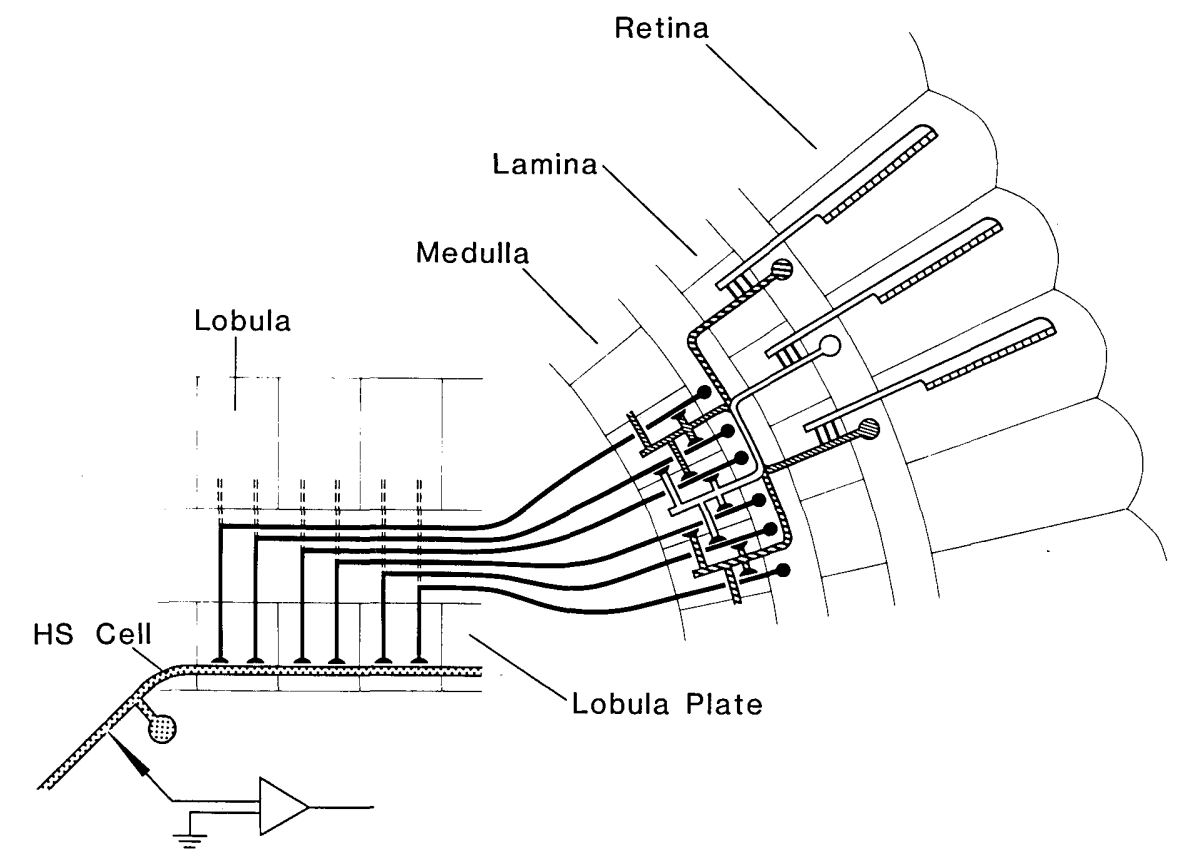

Fig. 1. Schematic diagram of the fly's visual system. Photoreceptors are located in the retina in separate ommatidia. They project retinotopically through several ganglia (lamina, medulla) into the lobula complex, which is subdivided into the anterior lobula and the posterior lobula plate. In the lobula plate, local movement information is spatially integrated in some way by giant tangential cells. One of these cells (the HS cell) was used in the present study to monitor the response of the movement-detection system. Note that the specific structure of different cell types shown here, including the connectivity at different stages, is arbitrary. 

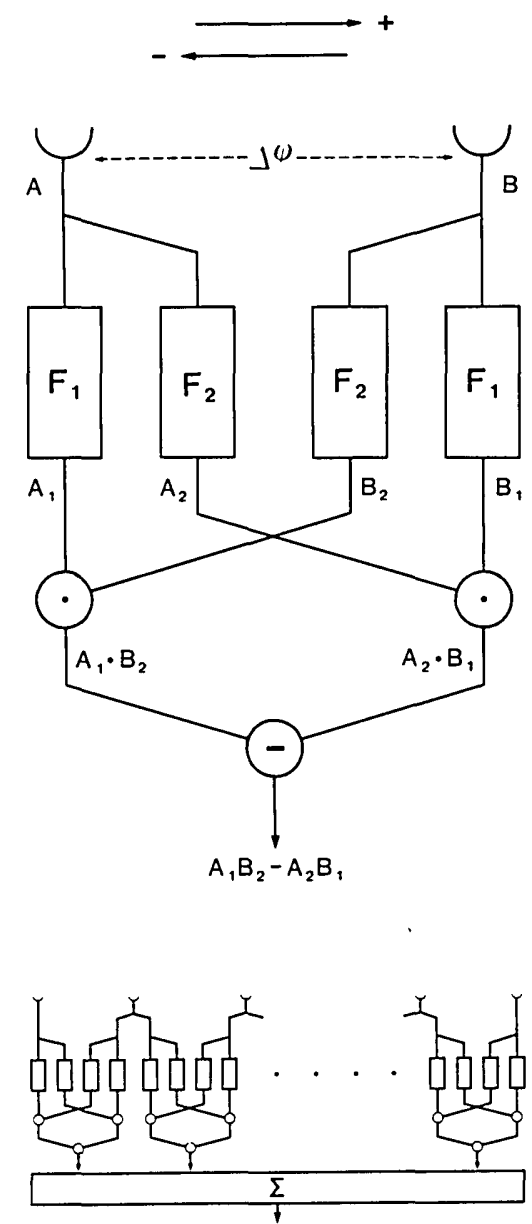

Fig. 2. General scheme of a movement detector of the correlation type. The input signals $A$ and $B$ are transmitted linearly by the receptors. Then they pass through linear filters $F_{1}$ and $F_{2}$. In the next step the filtered signals $A_{1}$ and $B_{2}$ are multiplied together. This procedure is repeated in the mirror-symmetrical subunit of the detector with the signals $A_{2}$ and $B_{1}$. The products $A_{1} B_{2}$ and $A_{2} B_{1}$ are subtracted from each other. In this way one results in a directionally selective movement detector that responds to leftward and rightward motion with the same strength but with the opposite sign. A retinotopic array of such movement detectors (schematically drawn) is shown below. Spatial integration is achieved by simply summing $(\Sigma)$ the output signals of all movement detectors. Both the steady-state and the transient responses of an array of detectors are calculated in Appendixes A and B for periodic input functions.

moving from back to front, the HS cells show a pronounced hyperpolarization. In contrast, front-to-back movement leads to a depolarization of the cell, upon which are superimposed small spikelike potentials. ${ }^{24}$ The graded depolarizations and hyperpolarizations of the HS cells reflect the summed synaptic potentials elicited by their presynaptic retinotopic input elements. Therefore in the experiments shown here we stimulated these cells by motion from back to front and, consequently, induced only graded potential changes. Responses obtained by stimulus motion from front to back are qualitatively similar but nevertheless are harder to interpret. The smooth postsynaptic membrane potential changes are then contaminated by action potentials. However, when taking spike-frequency histograms of the responses of other motion-sensitive cells, we obtain essentially the same results as shown here.
Our theoretical analysis of the motion-dependent responses is based on an array of movement detectors of the correlation type. An individual movement detector consists of two mirror-symmetrical subunits that share their two neighboring input channels. In the rather general form of the movement-detector model shown in Fig. 2 (upper schematic) the two input signals of each subunit pass through linear filters $F_{1}$ and $F_{2}$. These filters have to differ in an appropriate way in order for the detector to be directionally selective for motion and have usually been assumed to represent low-pass filters with a different time constant. ${ }^{1,7}$ In each subunit the filtered signal from one retinal location is multiplied with the filtered signal originating from the neighboring input channel. The final movement-detector output is then given by the difference of the two subunit outputs. Because the HS cells in the fly's brain pool the movement information from a considerable portion of the visual field, an array of movement detectors rather than an individual one will be the basis for our theoretical predictions. For convenience, we use a one-dimensional array of detectors, as is shown in Fig. 2 (lower schematic). The spatially integrated movement-detector output under both steady-state and transient conditions is calculated in Appendixes $A$ and $B$.

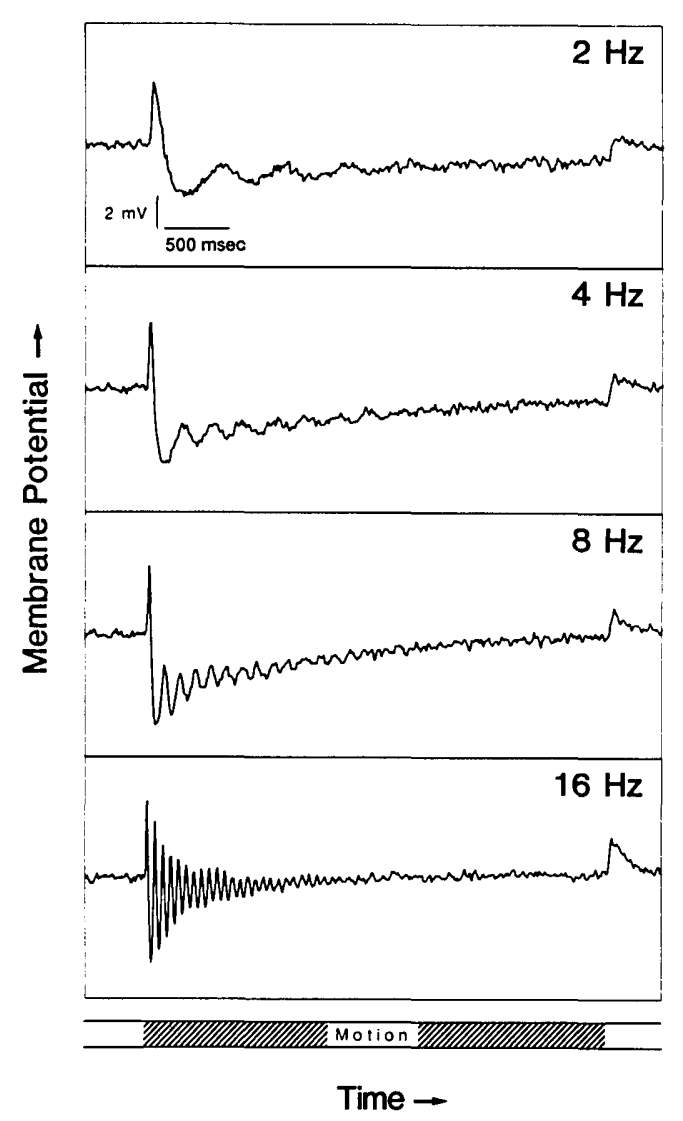

Fig. 3. Intracellular responses of the HS cell to sine-wave gratings (contrast, 0.1) moving back to front at different temporal frequencies, as indicated in the figure. Note that initially the cell's membrane potential is modulated with the pattern's temporal frequency before reaching a steady-state level. The data are averaged from two flies, each stimulated 20 times with the entire stimulation program of all five temporal frequencies. 


\section{A. Transient Responses to Pattern Motion}

To study the transient responses of the HS cells to the onset of motion, we started a sine-wave grating moving abruptly from back to front with a constant speed after it was at rest for some time. This is shown in Fig. 3 for five different temporal frequencies ranging from 2 to $16 \mathrm{~Hz}$ (from top to bottom); the temporal frequency is the ratio of the velocity to the spatial wavelength of the pattern. Each response trace represents an average of 40 response cycles from the same two flies. In this experiment a relatively low contrast was chosen in order to prevent nonlinearities in the input channels of the movement detector from affecting the response (see below). During pattern movement from back to front, the cell hyperpolarizes on average. After the onset of motion the membrane potential oscillates with a frequency that exactly reflects the pattern's temporal frequency. The membrane potential oscillations may be preceded by a brief depolarizing deflection, which, however, cannot consistently be found in all preparations (see below). At high oscillation frequencies (16 Hz in Fig. 3), the membrane potential can even cross the resting potential during its oscillatory phase and, in this way, can transiently depolarize the cell in response to back-to-front motion. The oscillation amplitude gradually decreases with time. After approximately 1-2 sec the cell's potential reaches a steady-state plateau that is more or less stable during the rest of the stimulation time. For the low pattern contrast the steady-state response of the cell is rather small, and the hyperpolarizing transient-response peaks assume amplitudes close to the maximum response amplitudes, which can be obtained in a HS cell by back-to-front stimulation. Because the pattern position at the onset of motion was randomized in the different stimulus presentations, the oscillations of the membrane potential do not depend on the spatial phase of the pattern relative to the eye.

Can these transient responses of the HS cells to motion be accounted for on the basis of correlation-type movement detectors? To answer this question an input equivalent to that used in our experiment was fed into a retinotopic array of detectors. In the computer simulations shown in Fig. 4, as well as in the analytic formulation of the responses (Appendix $B$ ), first-order low-pass filters were used as filters $F_{1}$, and the filters $\mathrm{F}_{2}$ were omitted completely. The temporal frequency can then be given in units of the filter time constant, as is done in Fig. 4. When the pattern starts moving, the spatially integrated movement-detector response reflects the corresponding response of the HS cells in many respects. It shows oscillations with a decreasing amplitude until it settles at its steady-state level. Again, the temporal frequency of the stimulus is reflected in the oscillation frequency of the response. The time constant of the exponential decay of the modulation amplitude represents the time constant of $F_{1}$ if there is only one detector filter (see Appen$\operatorname{dix} B$ ). Simulations with various other filter combinations of $F_{1}$ and $F_{2}$ have shown that the general phenomenon of response oscillations after the onset of motion are robust against the particular choice of the movement-detector filters. From the similarity between the experimental data (Fig. 3) and the computer simulations (Fig. 4), we can conclude that not only time-averaged characteristics but also specific dynamic properties of the cell's response to move-

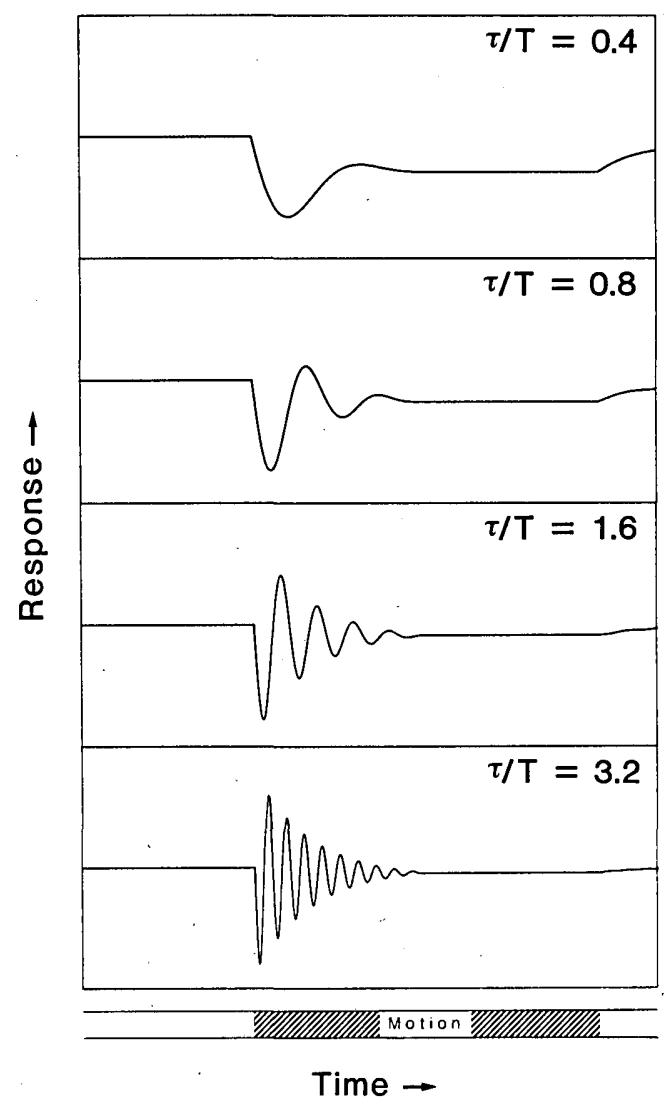

Fig. 4. Computer simulation of the responses of an array of movement detectors stimulated by equivalent inputs as in the experiment shown in Fig. 3. The detector signal can be seen to oscillate with the pattern's temporal frequency (given here in units of the filter time constant). Note the close similarity between the computer simulations and the experimental data of Fig. 3.

ment stimuli can be described well by a movement-detector model of the correlation type.

It should be noted, however, that there are two features in the cellular responses that cannot be accounted for by the model as used here and by all other variants tested so far. First, in the electrophysiologically recorded responses the membrane potential oscillations may be preceded by a brief depolarizing deflection. This initial deflection, which is opposite that of the steady-state response, was found only in response to motion from back to front. There are, however, many examples in which it does not occur and, thus, does not represent a necessary by-product of the fly's movementdetection' system. Moreover, when the cell was stimulated with front-to-back motion, no hyperpolarizing deflections were observed (data not shown). Second, when the pattern stops moving, there is a transient depolarization of the HS cell, which cannot be found in the model simulations. Here the membrane potential declines to its resting level with a time constant that reflects the time constant of the movement-detector filter. At present we cannot decide whether the latter discrepancy between model simulations and experimental results is a by-product of general physiological properties of neurons or whether it reflects a specific functional property of the fly's motion-detection system that has not been taken into account so far in our model. 


\section{B. Contrast Dependence of the Movement-Detector Responses}

The dynamic response properties of movement detectors do not only provide information about the performance of the system. In comparison with the steady-state responses they also can be used as a tool to localize additional nonlinearities in the motion-detection pathway. Here we analyze the contrast dependence of the fly movement-detection system under both transient and steady-state conditions. The experimental procedure was the same as described above, with the exception that the pattern contrast was varied between 0.05 and 0.5 at two different temporal frequencies ( 1 and $10 \mathrm{~Hz}$ ). Each data point is an average of 37 stimulus presentations obtained from the same sample of nine different flies. As is indicated schematically in the insets of Fig. 5, the maximum hyperpolarizing response peak after the onset of motion and the mean steady-state response amplitude (between 2.6 and $3.6 \mathrm{sec}$ after the onset of motion) were evaluated separately from the time-dependent traces.

Both the steady-state and the hyperpolarizing peak responses show a highly nonlinear dependence on pattern con-

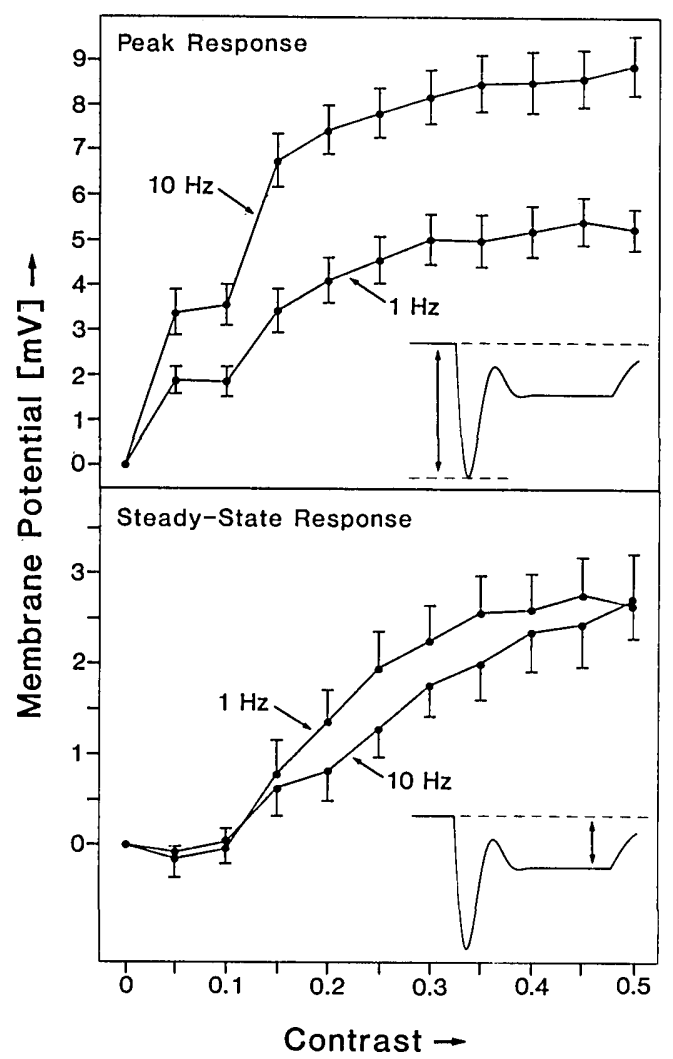

Fig. 5. Average response amplitudes of the HS cell to sine-wave gratings of different contrast moving back to front at two different temporal frequencies ( 1 and $10 \mathrm{~Hz}$ ). Schematic time-dependent response traces are shown as insets to illustrate how peak and steady-state responses were determined. The average peak and steady-state response amplitudes are shown as functions of the pattern contrast. The bars indicate the standard error of the mean. The data are averages from 9 flies and 36 repetitive presentations of the entire stimulus program. There is a difference in the contrast dependence of both peak response curves, of peak and steady-state responses to the same frequency, and of both steady-state response curves. Note the different ordinate scales used in the two diagrams. trast. From the detector theory (see Appendixes A and B) the responses are expected to increase as a quadratic function with pattern contrast. In a biological system this is, of course, possible only in a limited range. The responses cannot increase indefinitely, but they approach a final maximum response level. Although the amplitude of the peak responses reaches $80 \%$ of its maximum value at contrasts between 0.15 and 0.20 , the steady-state responses reach their final level only at higher contrasts. It should be noted that the contrast dependences of the transient and steady-state responses are plotted at different scales. Thus, for the temporal frequencies used here, the final amplitudes of the hyperpolarizing transient responses are considerably higher than their corresponding steady-state counterparts. Only for low temporal frequencies do the peak and steady-state responses assume the same amplitudes. Moreover, the transient response at the different temporal frequencies approaches different final response levels. At $1 \mathrm{~Hz}$ it has less than two thirds of the amplitude obtained at $10 \mathrm{~Hz}$. The shape of the contrast-dependence curves is, however, the same for both temporal frequencies; the two curves differ only by a factor. The situation found for the steady-state responses is much different. Here the responses obtained for a temporal frequency of $1 \mathrm{~Hz}$ reach their final response level at lower contrasts than do the ones for $10 \mathrm{~Hz}$. At high contrasts, both responses reach approximately the same amplitude, but the two curves differ to some degree with respect to their shape: unlike the transient peak responses, they are not just a multiple of each other. Similar contrast dependences for different temporal frequencies have also been found at the behavioral level in the optomotor yaw torque response of the housefly Musca domestica. ${ }^{27}$

The specific contrast dependence of both transient and steady-state responses can, in principle, be accounted for by simple saturation nonlinearities. Where these nonlinearities might be located in the movement-detection system can be inferred from the findings shown in Fig. 5. This is possible because the sequence of linear and nonlinear elements in a pathway can strongly affect the output of the network. Saturation at the level of the HS cell, i.e., after spatial integration has been accomplished, cannot explain the experimentally determined contrast dependences of the transient and steady-state responses, because in this case the initial transient as well as the steady-state response should approach a common response level irrespective of the temporal frequency. A saturation characteristic before spatial integration at the output of the individual movement detectors can be dismissed for similar reasons. Hence the specific dependence of the movement-detector response on contrast is likely to be due to saturation nonlinearities somewhere at a more peripheral stage.

Before these saturation nonlinearities can be localized, we have to consider some general aspects of saturation characteristics. Let us assume that there is an element in the motion-detection pathway, such as a nerve cell, the activity of which can be modulated in both directions about a resting level. Let us further assume that the element saturates for both increments and decrements of its response. Then we obtain a sigmoidal input-output response characteristic (Figs. 6a and 6b). When a periodic input signal passes through this type of nonlinearity, both the modulation am- 


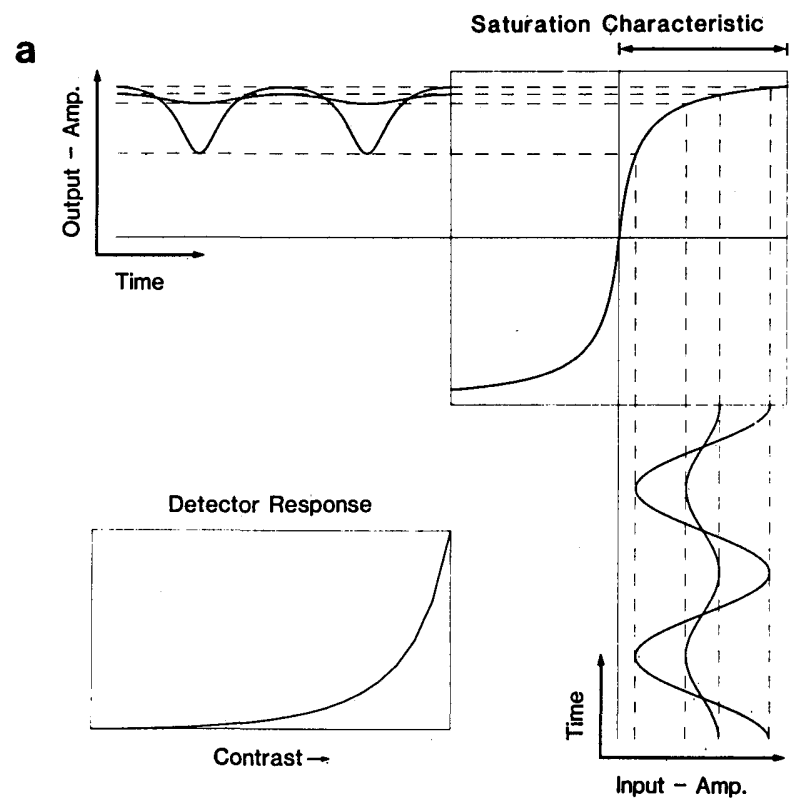

b

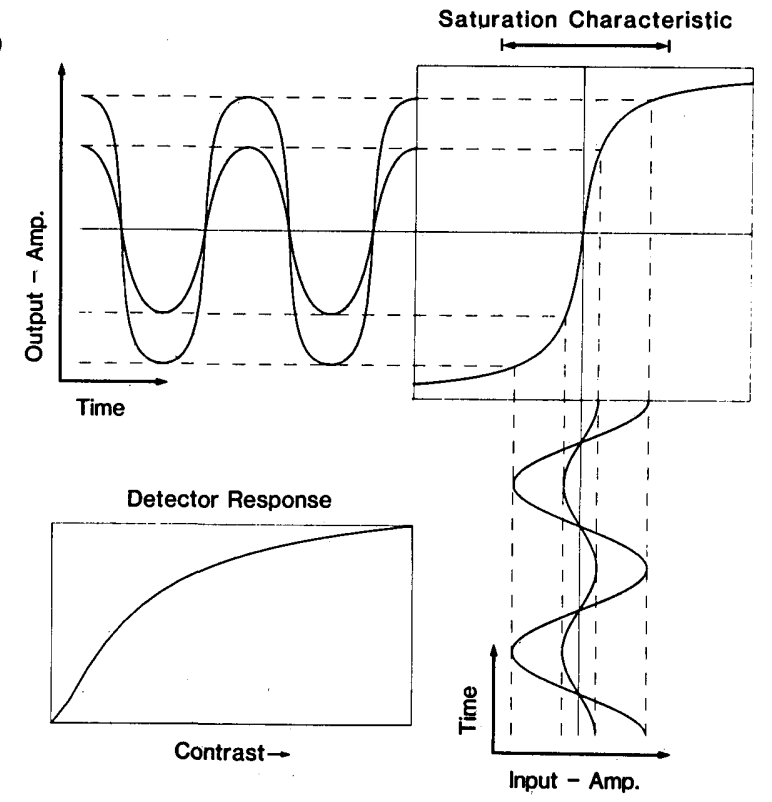

Fig. 6. Consequences of a saturation characteristic for contrast coding of a movement detector. A sinusoidal input signal (shown below the input-output characteristic) passes an element that saturates for both increments and decrements of its response. The resulting output signals are shown at the left of the input-output characteristic. a, If the signal is proportional to the light intensity received by the eye, it can be modulated only between twice its mean amplitude and the zero level. This range is indicated by the doubleheaded arrow above the saturation characteristic. As can be seen by comparing the input and the output modulations, the signal does not saturate at all under these conditions. Consequently the resulting movement-detector response (see inset) will increase steeply with increasing modulation amplitude of the input signal. b, If the mean luminance is removed in some way, the input signal is zero symmetrical and, thus, modulated about the steep-slope part of the input-output characteristic. In this case small modulation amplitudes will be transmitted with a high gain, whereas larger ones will approach a saturation level. Accordingly, the movement-detector response shown in the inset also saturates for larger modulation plitude and the shape of the signal may be affected. These changes strongly depend on the extent to which the mean luminance is represented in the input signal. Two extreme cases are illustrated in Fig. 6. When the signal is proportional to the light intensity, it can assume only positive values. By passing through the nonlinearity, the signal, therefore, will not saturate. As a consequence, the resulting movement-detector output (insets in Fig. 6) will increase steeply with an increasing modulation amplitude. A saturationlike contrast dependence of the movement-detector output can be achieved, except by a simple output saturation, only if the mean level of the input signal is eliminated in some way before passing through the sigmoidal nonlinearity (see Fig. 6b). Because we find this kind of saturationlike phenomenon in our experimental results (Fig. 5), we have to propose that the background intensity be eliminated at least to some extent from the movement-detector input signals before being processed further. Because saturation phenomena are almost unavoidable in neuronal elements, the elimination of the mean light intensity seems to be an essential precondition for a meaningful contrast coding by the movement-detection system.

Saturation at the level of both the HS cell and the individual movement-detector output has already been discarded as an explanation of the experimental results. Therefore, in the motion-detector model of Fig. 2, saturation nonlineari-

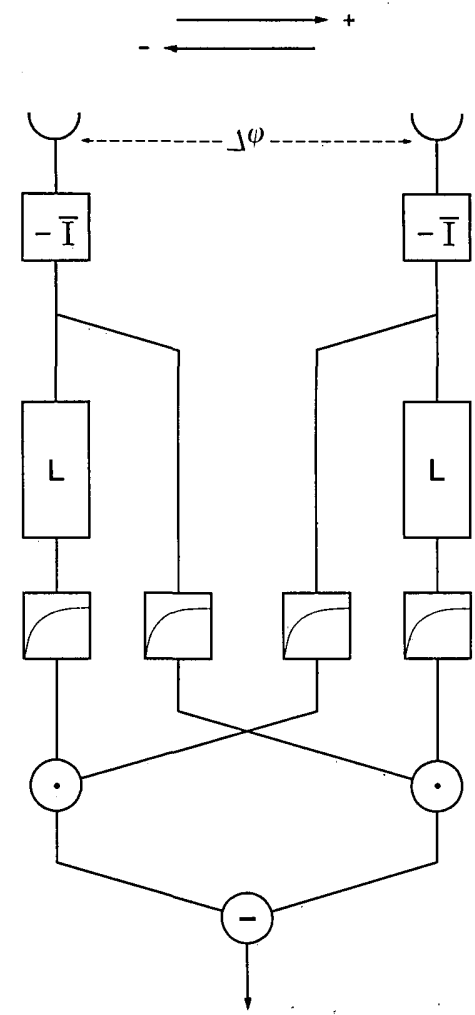

Fig. 7. Elaborated version of the model proposed to underlie the evaluation of movement in flies. The general scheme of a movement detector of the correlation type (see Fig. 2) is modified in three ways: (i) the mean luminance is subtracted from the input signals; (ii) saturation characteristics are inserted into both branches of the two movement-detector subunits, (iii) the filter $\mathrm{F}_{2}$ of the general movement-detector scheme shown in Fig. 2 is omitted. The filter $\mathrm{F}_{1}$ is specified here as low-pass filter $\mathrm{L}$. 


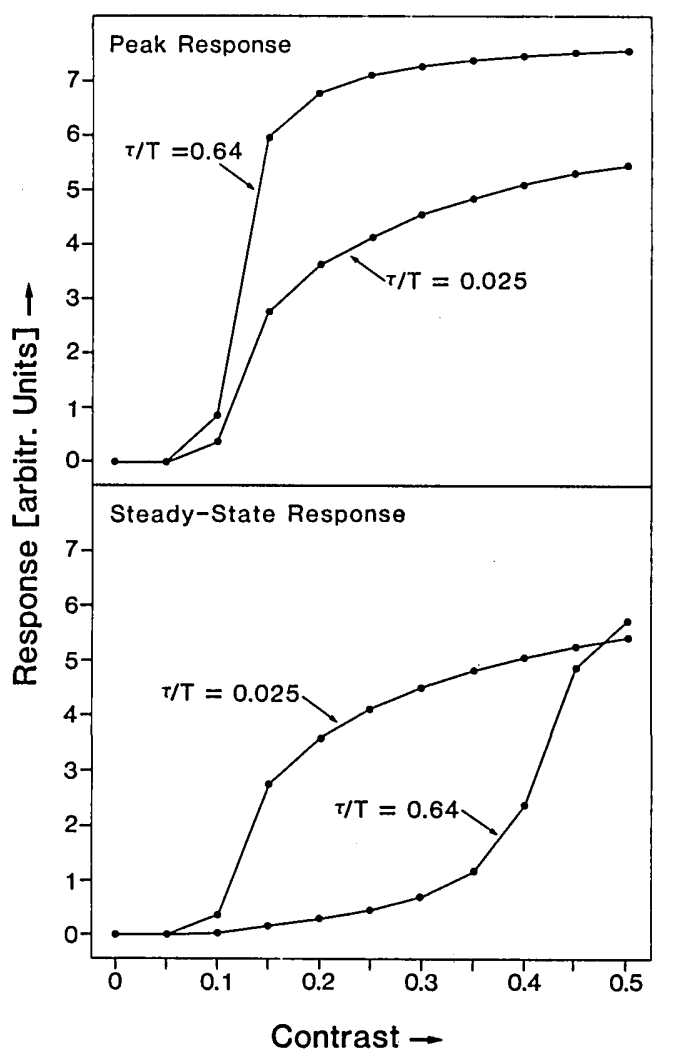

Fig. 8. Contrast dependence of a one-dimensional array of movement detectors as elaborated in Fig. 7. Filter L, a first-order lowpass filter, was chosen. The peak and steady-state responses to the outset of motion were evaluated for two different temporal frequencies as the corresponding experimental data of Fig. 5. The temporal frequency of the stimulus is given in units of the time constant of the movement-detector filter. The computer simulations of the model account for the qualitative features of the corresponding experimentally determined responses (compare with Fig. 5).

ties can be located only in the input channels to the detector or after the low-pass filter. In Fig. 7 only the latter possibility is illustrated, as saturation in the input channels cannot explain our data (see below). This specific version of the movement-detector model represents perhaps the simplest version that can account for the principal features of our data and is derived from the more general scheme of a movement detector of the correlation type (see Fig. 2) by three alterations: (i) the filters $F_{2}$ proved to be unnecessary and, therefore, are omitted from the model; (ii) the mean luminance is eliminated in some way from the input signals of the movement detector (in our model it is simply subtracted; see Subsection 4.B.1); (iii) saturation characteristics are inserted into both branches of each detector subunit; in the branch that contains the low-pass filter the nonlinearity is inserted after the filter. It should be noted that our computer simulations were used only to find a kind of minimal model version that was sufficient to account for the principal features of the experimental results qualitatively rather than to fit the data as closely as possible. A closer fit certainly would have been possible if the model had been elaborated further (see below).

Computer simulations based on this version of the detector model are displayed in Fig. 8. The contrast dependence of both the initial negative response peaks after onset of motion and the final steady-state response plateau are shown here for two different temporal frequencies. Again, as in Fig. 4, the temporal frequencies are given in units of the time constant of the movement-detector filter. Both the transient and the steady-state responses approach different saturation levels, which, in addition, depend on the temporal frequency of the stimulus. For all contrasts the levels of the transient-response peaks increase with increasing temporal frequencies, which is in accordance with our experimental results (compare the upper parts of Figs. 5 and 8). This effect is even more pronounced if the filter $F_{2}$ is not omitted but is replaced by a high-pass filter with an appropriate time constant. The steady-state-model responses depend on contrast in quite a different way (Fig. 8, bottom). Most importantly, the shape of the contrast-dependence characteristics depends on the temporal frequency of the stimulus. This is, at least qualitatively, in accordance with the experimental data as described above (compare the lower parts of Figs. 5 and 8). A different contrast dependence of the steady-state responses at different temporal frequencies is not obtained when the saturation characteristic is inserted before the low-pass filter. The resulting curves for the different temporal frequencies are almost parallel in this case. The quantitative discrepancies between the model simulations and the experimental results are due to the fact that the movement-detector time constant is not a constant, as was assumed in the simulations, but depends on the stimulus conditions (see Section 4). ${ }^{28,29}$ If the stimulus dependence of the time constant is taken into account, the difference between the contrast-dependence characteristics obtained at different temporal frequencies becomes smaller, as in the experimentally determined curves, and may even disappear totally. Nevertheless, the rather simple model shown in Fig. 7 is certainly sufficient to describe, at least in a first approximation, the performance of the fly's movementdetection system under both transient and steady-state conditions with respect to its contrast dependence.

\section{DISCUSSION}

Theoretical as well as experimental studies of the visual system of insects have played a decisive role in the conceptualization of the basic principles underlying movement detection. 1,2 The movement detector of the correlation type that has emerged from these studies could subsequently be applied successfully to certain aspects of motion vision in humans. ${ }^{10-14}$ Thus the movement-detection system of the fly can be regarded as a biological model system for experimentally studying the properties of movement detectors of the correlation type. The following features are essential for the adequate performance of this movement-detection scheme: It has two spatially displaced input channels that feed their signals into two mirror-symmetrical subunits. In each subunit the signals from the neighboring input stages are multilied after they have been delayed in some way with respect to one other. Finally, the outputs of the two subunits have to be subtracted in order to make the movement detector directionally selective and its output independent of the mean intensity of the stimulus pattern (see Appendix A). The subtraction stage may be omitted if special combinations of filters are chosen in both branches of the detector subunits. A combination of a low-pass and a high-pass filter 
of the same order and with the same time constants represents such a special possibility. ${ }^{30}$

What properties of the movement detector are a consequence of these essential components and thus are inevitable on the basis of this detection mechanism? There are two main consequences. The first concerns the possibility of response transients. Depending on the stimulus motion, the detector cannot always be assumed to operate under steady-state conditions, and the response may then deviate considerably from being proportional to the stimulus velocity. ${ }^{20}$ The second consequence concerns a well-known property of movement detectors of the correlation type. The detector signal does not unambiguously reflect the stimulus velocity, even under steady-state conditions. Instead, it depends on the textural properties of the stimulus pattern, such as its spatial-frequency content and its contrast.1,2,19,31 This dependence of movement perception on the properties of the pattern is not a specific feature of insects but is also well established in other systems, such as in human vision. ${ }^{32-38}$ Here we have studied the contrast dependence of the detector response and how the problem of an ambiguous movement-detector output signal can be alleviated by elaborating the detection scheme in a simple and physiologically plausible way.

\section{A. Transients to the Onset of Motion}

The onset of motion of a stimulus pattern represents a special situation in which the movement-detector theory as formulated to account for the steady-state responses cannot be applied. This stimulus condition was chosen in our experiments because it is in common use in psychophysical and electrophysiological experiments, and the detector response can be derived by simple calculation (see Appendix B). We used the visually induced membrane potential changes of the large-field HS cells ${ }^{23}$ as our indicator for the performance of a biological movement-detection system. Three features of the HS cells' transient responses to periodic onedimensional patterns are particularly interesting. First, the membrane potential oscillates with the temporal frequency of the stimulus pattern, and only after a while does it reach a time-independent steady-state level. Second, during the transition phase the response profiles may cross the zero response level and then signal motion in the wrong direction. Third, the instantaneous peak response amplitudes can be much larger than the final steady-state level. These response characteristics of the HS cells can be easily, at least qualitatively, interpreted as consequences of the intrinsic properties of an array of movement detectors of the correlation type.

The modulations in the response transients are found only if the stimulus pattern is periodic. In the case of patterns with a random texture, the response is expected to peak transiently immediately after the onset of motion and then to decay exponentially to the steady-state level. This is just what can be found in directionally selective large-field neurons in the fly, but it has been interpreted as the result of some kind of adaptation phenomenon. ${ }^{39}$ However, from the present results it seems to be quite certain that transient movement-detector properties play a decisive role in shaping these experimentally determined response transients of the fly's large-field neuron.

The time constant of decay of the transient responses both to periodic and to random patterns should reflect the time constant of the movement-detector filters (see Appendix B). This, however, is true only so long as no other processes with time constants in the critical range interfere with the movement-detector responses. One such process is the so-called adaptation of the movement-detector time constant. ${ }^{28,29}$ This means, depending on both the temporal frequency and the contrast of the stimulus pattern, that the detector time constant continually decreases after onset of motion until it reaches its final steady-state level. The main decrement of the time constant is found within the first $2 \mathrm{sec}$ after onset of motion. ${ }^{28}$ In this way the time constant can assume values within a range of approximately $500-5$ msec. ${ }^{20,28,29}$ The variable time constants of the fly movement detectors imply that it is not possible to interprete the time constant of decay of the response transient to the onset of motion in a straightforward way. Instead, it reflects both the adapation process of the detector time constant ansd its instantaneous value.

Because the transient and the steady-state responses of a movement-detector array may differ considerably, the transient responses should be taken into account in interpreting experimental data obtained under transient stimulus conditions. In this way the characteristic response transients that have now been found experimentally were predicted in a behavioral analysis on the processing of movement information by the landing system of the housefly. ${ }^{21}$

By disregarding the importance of the response transients of the movement-detection system, Eckert and Hamdorf 40 drew incorrect conclusions with respect to the potential neuronal substrate of the fly's landing system. They tried to relate the optimum temporal frequency of a movementsensitive visual interneuron as obtained under steady-state conditions to the optimum temporal frequency of the landing response. However, the optimum temporal frequency of the landing response is significantly affected by transient movement responses. ${ }^{21}$

In an electrophysiological study of another motion-sensitive visual interneuron of the fly, ${ }^{41,42}$ transient patterns of spike activity have been found in the response to the onset of motion. These patterns are virtually identical to the intracellularly recorded membrane potential oscillations as measured in the present study. These phenomena are interpreted, however, without consideration of movement-detector theory. Instead, the measured transient phenomena are accounted for in terms of afterimagelike effects, which are assumed to reduce the sensitivity of the input channels to the interneuron locally. ${ }^{41,42}$ From the close similarity of the transient phenomena found by Maddess ${ }^{41,42}$ and from our own findings, we would like to suggest that both findings simply reflect the intrinsic properties of the motion detectors forming the input to these cells. This inference is further supported by other, more complex experimental results of Maddess, ${ }^{41,42}$ which can be accounted for equally well by the movement-detector theory. For instance, the time course of the transient responses to moving patterns strongly depends on the textural properties of the stationary stimulus that is seen by the animal before the onset of motion. If both the moving and the stationary patterns are periodic gratings, the modulation amplitude of the initial oscillations of the response decreases with a decreasing contrast of the stationary grating.

The two examples discussed above clearly demonstrate 
that it is worthwhile to take movement-detector theory into account when we interpret complex motion-dependent experimental data. Moreover, the existence of transient responses should be considered if respective stimuli are used. Then the data, in general, cannot be explained purely on the basis of the steady-state detector theory. This might also be important when we interpret psychophysical data because transient movement stimulation is in common use there and often leads to surprising results. ${ }^{43}$

\section{B. Nonessential Nonlinearities in the Movement- Detection System}

The general scheme of a correlation-type movement detector is elaborated here in two ways in order to account for our experimental data on the fly visual system. First, it is proposed that the mean background luminance be subtracted from the detector input signals. Second, saturation nonlinearities are inserted into both branches of the two detector subunits (see Fig. 7). Although both elaborations are not essential in order for the detector to work, they endow the detector with properties that might be beneficial for its special purposes.

\section{Elimination of the Background Luminance}

The elimination of the mean background luminance turns out to be necessary if the detector input channels contain saturationlike nonlinearities, which cannot easily be avoided in any biological system. Elimination of the background luminance in the input channels to the movement detector, at least in the form shown in Figs. 2 and 7, does not affect the detector output otherwise and, consequently, earlier conclusions on its properties because the final detector output is independent of the mean luminance anyway (see Appendix A). Eliminating the mean luminance peripherally might even be advantageous for a movement detector that has to be implemented by biological or technical hardware. If the mean luminance were not eliminated in the input channels, the detector output signal would usually be small relative to the signals of the two detector subunits that are to be subtracted. Thus a movement detector made of neuronal components might be confronted with serious problems in signal-to-noise ratio.

In our elaboration of the movement-detector model (see Fig. 7) the background luminance is eliminated simply by subtracting it from the local light-intensity signals. Temporal high-pass filtering of the movement-detector input signals would be another simple means to eliminate background luminance. However, because of the experimental results of Maddess, ${ }^{41,42}$ this possibility can be discarded. As was discussed above, the time course of the transient responses to the onset of motion is strongly affected by the texture of the stationary pattern presented before the movemet stimulus. A linear temporal high-pass filter would, of course, represent all stationary patterns in the same way as do uniform fields.

It is interesting to note that in both vertebrates and invertebrates the mean background luminance is removed to a high degree at processing stages peripheral to movement detection, although most likely by different mechanisms. In the vertebrate retina this is accomplished by the centersurround organization of the ganglion cells' receptive fields. ${ }^{44}$ For this reason, spatial band-pass filters are usual- ly inserted into the input channels of those movement-detection models used to explain motion vision in humans. ${ }^{12,16}$ In the fly's visual system the background luminance is removed in the first visual ganglion, the lamina (see Fig. 1), by a mechanism that is not completely understood so far. At the synapses between the retinal receptor cells and the firstorder interneurons, the background luminance is eliminated by a kind of subtractive inhibition, while at the same time the contrast signal is expanded to fill the dynamic range of the interneuron. However much the mean intensity varies, the strength of inhibition is always adjusted to keep the cell's response centered on the band of contrast signals. ${ }^{45,46}$ Hence the peripheral processing of visual information appears to be excellently adapted to the requirements of motion detection.

\section{Saturation Nonlinearities in the Movement-Detection System and Their Functional Significance}

The initial hyperpolarizing transients of the HS-cell response to the onset of back-to-front motion have been found to be relatively independent of contrast, at least for contrasts above 0.15 , whereas their amplitude increases with increasing pattern velocity. The steady-state responses are different in this respect. They increase, depending on the temporal frequency of the stimulus, up to much higher contrasts and, therefore, are similar in this regard to corresponding behavioral data ${ }^{31,47}$ and electrophysiological findings on another motion-sensitive large-field neuron in the fly. ${ }^{48}$ The simplest way to account for these results is to assume saturation nonlinearities before multiplication of the movement-detector input signals. Interestingly, when studying apparent motion phenomena in flies, Bülthoff and Götz ${ }^{49}$ also proposed a saturation nonlinearity in the movement-detector input channels. On the basis of their data, however, they could not decide whether the saturation characteristic is located before or after filtering of the input signals.

It is suggested by our findings for the contrast dependence of the HS-cell responses that the transient-response peaks are relatively invariant against variations in pattern contrast but, at the same time, represent (at least qualitatively) the temporal frequency of the pattern. On the other hand, the steady-state responses are much more ambiguous in this respect and do not provide reliable estimates of the temporal frequency of the stimulus pattern. It should be noted, however, that the steady-state response does not unambiguously code for the temporal frequency anyway because, as was explained above, the time constant of the movement-detector filter adapts when the eye is exposed to motion, even for only a fraction of a second. ${ }^{28,29}$ This adaptation of the time constant results in a considerably expanded range of temporal frequencies that lead to large response amplitudes at the movement-detector output. ${ }^{26,50,51}$

The functional significance of the differences between the transient and steady-state responses can be assessed appropriately only if we take into account the conditions under which the particular movement-detection system has to operate. We are in the fortunate situation that there is detailed knowledge about visual orientation behavior of the fly both in free flight and under laboratory conditions..$^{3,22,52}$ In visual orientation flies exploit motion information for different purposes. For instance, specific feedback control sys- 
tems enable the fly to stabilize its flight course against internal and external disturbances and to turn toward objects that move relative to the retina. In optomotor flight-course stabilization only slow changes of retinal large-field motion are compensated for. ${ }^{53}$ Therefore the movement-detection system can be assumed to operate under steady-state conditions during course stabilization. The feedback control system, however, is not required to rely on unambiguous velocity measurements under these dynamic conditions. On the other hand, brief velocity transients occur in free-flying houseflies during active turns. ${ }^{54}$ When these transients result from relatively small objects, they may induce turns toward these objects, ${ }^{53}$ such as in pursuit of other flies..$^{52,55}$ Under these conditions the inevitable time constants in the feedback loop of the underlying control system can be assumed to be of the same order of magnitude as the duration of the movement transients; therefore corrective fine tuning of a turn after it has been induced might not be possible. This suggests that an unambiguous representation of velocity during motion transients might be useful. With respect to pattern contrast, this invariance is more or less realized. At the same time, faster movements result in larger response transients of the movement-detection system.

As a consequence of an adaptation of the movement-detector time constant to its optimal value, the response transients are predicted to signal correctly, at least in a certain velocity range, both increments and decrements of pattern velocity from its mean level. Moreover, a given, brief relative deviation from the mean velocity is expected, in a first approximation, to lead to response transients of always the same amplitude, irrespective of the mean velocity. It is interesting to note that this was actually found experimentally, at least to some degree, in a motion-sensitive interneuron in the fly's visual system. ${ }^{39}$ It should be emphasized that this kind of coding of velocity contrast ${ }^{39}$ can be accounted for simply by the intrinsic properties of the movement detector of the correlation type together with the assumption of a detector time constant that appropriately adapts to the stimulus velocity.

\section{Other Movement-Detection Schemes}

In this study we concentrated on a comparison of experimental data with the predictions of the so-called correlationtype movement detector. This scheme specifies the different computations underlying movement detection in a formal way and, therefore, represents an algorithmic model. The advantage of this level of description is that it allows us to account for the operations independently of the specific hardware by which they are implemented. This is of particular importance if we want to compare motion detection in different biological and technical systems because a given algorithm can be realized on the hardware level in completely different ways.

There are other algorithmic models that have been proposed to underly motion detection in biological systems. One class of detectors are the so-called spatiotemporal energy models. ${ }^{16}$ Although they differ considerably from the correlation-type movement detector with respect to their internal structure, both motion-detection schemes are, under certain assumptions, mathematically equivalent at their output. ${ }^{15,16}$ Therefore they cannot be distinguished on the basis of the present experiments. Another class of models, the so-called gradient schemes, ${ }^{56,57}$ cannot account for response transients as reported here because the output of this model, at least in its mathematically ideal form, should always be proportional to pattern velocity. This is in accordance with earlier experimental results on steady-state responses that exclude the gradient scheme for motion vision in insects. ${ }^{3}$

Of course, some components of the fly's movement-detection system as proposed in this study (Fig. 7) can be accounted for also in cellular terms. The likely cellular bases of the elimination of the mean luminance from the light-intensity distribution as received by the eye and the saturation nonlinearities in the detector input channels were discussed in previous sections. Cellular models were also proposed for the essential multiplicationlike interaction of the two movement-detector input channels. The so-called shunting inhibition model ${ }^{58,59}$ represents the most widely discussed possibility. There is some experimental evidence that this cellular interaction scheme plays a role in motion detection in the fly visual system. ${ }^{60}$ Whether this type of cellular model can account for the multiplicationlike interaction is currently being investigated.

\section{APPENDIX A: STEADY-STATE DETECTOR RESPONSE}

The definitions used for all calculations are shown in Table 1.

Moving a sine grating across a one-dimensional array of movement detectors (see Fig. 2) with a constant velocity leads to the following input signals at each detector input channel:

$$
\begin{aligned}
& A=I+\Delta I \sin (\omega t+\varphi) \\
& B=I+\Delta I \sin [\omega(t-\Delta t)+\varphi] .
\end{aligned}
$$

With $\Delta t=\Delta \varphi / w$ and $\omega=2 \pi w / \lambda, B$ becomes

$$
B=I+\Delta I \sin [\omega t+\varphi-2 \pi \Delta \varphi / \lambda] .
$$

The output functions of the linear filters are then

$$
\begin{aligned}
& A_{1}(t)=\mathrm{F}_{1}(0) I+\mathrm{F}_{1}(\omega) \Delta I \sin \left[\omega t+\varphi+\phi_{1}(\omega)\right], \\
& A_{2}(t)=\mathrm{F}_{2}(0) I+\mathrm{F}_{2}(\omega) \Delta I \sin \left[\omega t+\varphi+\phi_{2}(\omega)\right], \\
& B_{1}(t)=\mathrm{F}_{1}(0) I+\mathrm{F}_{1}(\omega) \Delta I \sin \left[\omega t+\varphi+\phi_{1}(\omega)-2 \pi \Delta \varphi / \lambda\right]
\end{aligned}
$$

\section{Table 1. Definitions of All Parameters}

\begin{tabular}{ll}
\hline Parameter & \multicolumn{1}{c}{ Definition } \\
\hline$I$ & Mean luminance of the pattern \\
$\Delta I / I$ & Pattern contrast \\
$\lambda$ & Spatial wavelength of the pattern \\
$w$ & Angular velocity \\
$t$ & Time \\
$\varphi$ & Phase of the pattern at $t=0$ \\
$\Delta t$ & Time interval between the reception of the stimulus \\
& by the left and the right input channels \\
$\Delta \varphi$ & Angular distance between adjacent visual elements \\
$\omega$ & Angular frequency $=2 \pi \omega / \lambda$ \\
$F(\omega)$ & Amplitude-frequency function of a linear filter \\
$F(0)$ & Amplitude-frequency function for $\omega=0$ \\
$\phi(\omega)$ & Phase-frequency function of a filter \\
\hline
\end{tabular}




$$
B_{2}(t)=\mathrm{F}_{2}(0) I+\mathrm{F}_{2}(\omega) \Delta I \sin \left[\omega t+\varphi+\phi_{2}(\omega)-2 \pi \Delta \varphi / \lambda\right] .
$$

The spatially integrated steady-state response of a one-dimensional array of movement detectors is given by the expression (for details, see Ref. 1)

$$
R=\mathrm{F}_{1}(\omega) \mathrm{F}_{2}(\omega) \Delta I^{2} \sin \left[\phi_{1}(\omega)-\phi_{2}(\omega)\right] \sin (2 \pi \Delta \varphi / \lambda) .
$$

For the special case when the filter $F_{2}$ is omitted and $F_{1}$ is represented by a first-order low-pass filter with

$$
F_{1}(\omega)=\frac{1}{\left(1+\tau^{2} \omega^{2}\right)^{1 / 2}}, \quad \phi_{1}(\omega)=-\arctan (\tau \omega)
$$

$R$ becomes

$$
R=\Delta I^{2} \sin \left(2 \pi \frac{\Delta \varphi}{\lambda}\right) \frac{\tau \omega}{1+\tau^{2} \omega^{2}}
$$

\section{APPENDIX B: TRANSIENT DETECTOR RESPONSE TO THE ONSET OF MOTION}

For convenience filter $F_{2}$ will be omitted, and filter $F_{1}$ will be represented by a first-order low-pass filter. The input signals are then

$$
\begin{aligned}
& A= \begin{cases}I+\Delta I \sin (\varphi) & \text { for } t<0 \\
I+\Delta I \sin (\omega t+\varphi) & \text { for } t \geqslant 0\end{cases} \\
& B= \begin{cases}I+\Delta I \sin \left(\varphi-2 \pi \frac{\Delta \varphi}{\lambda}\right) & \text { for } t<0 \\
I+\Delta I \sin \left(\omega t+\varphi-2 \pi \frac{\Delta \varphi}{\lambda}\right) & \text { for } t \geqslant 0\end{cases}
\end{aligned}
$$

After convolution with the impulse response $g(t)=(1 / \tau)$ $\exp (-t / \tau)$ of a first-order low-pass filter with the time constant $\tau$, we obtain

$$
\begin{aligned}
A_{1}= & I+\Delta I \cos (\varphi) \frac{\tau}{1+\tau^{2} \omega^{2}} \\
& \times\left[\frac{1}{\tau} \sin (\omega t)-\omega \cos (\omega t)+\omega \exp \left(-\frac{t}{\tau}\right)\right] \\
& +\Delta I \sin (\varphi) \frac{\tau}{1+\tau^{2} \omega^{2}} \\
& \times\left[\frac{1}{\tau} \cos (\omega t)-\omega \sin (\omega t)-\frac{1}{\tau} \exp \left(-\frac{t}{\tau}\right)\right] \\
B_{1}= & +\Delta I \cos \left(\varphi-2 \pi \frac{\Delta \varphi}{\lambda}\right) \frac{\tau}{1+\tau^{2} \omega^{2}} \\
& \times\left[\frac{1}{\tau} \sin (\omega t)-\omega \cos (\omega t)+\omega \exp \left(-\frac{t}{\tau}\right)\right] \\
& +\Delta I \sin \left(\varphi-2 \pi \frac{\Delta \varphi}{\lambda}\right) \frac{\tau}{1+\tau^{2} \omega^{2}} \\
& \times\left[\frac{1}{\tau} \cos (\omega t)-\omega \sin (\omega t)-\frac{1}{\tau} \exp \left(-\frac{t}{\tau}\right)\right] .
\end{aligned}
$$

The response $R$ is again given by $A_{1} B-A B_{1}$, and after spatial integration we obtain

$$
\begin{aligned}
R(t)= & \Delta I^{2} \sin \left(2 \pi \frac{\Delta \varphi}{\lambda}\right) \frac{\tau \omega}{1+\tau^{2} \omega^{2}} \\
& -\Delta I^{2} \sin \left(2 \pi \frac{\Delta \varphi}{\lambda}\right) \frac{1}{\left(1+\tau^{2} \omega^{2}\right)^{1 / 2}} \\
& \times \sin [\omega t+\arctan (\tau \omega)] \exp \left(-\frac{t}{\tau}\right) .
\end{aligned}
$$

Thus the response of an array of movement detectors consists of two components. One component represents the steady-state solution (see Appendix A), and the other oscillates with the temporal frequency of the stimulus. The oscillation amplitude decays with the time constant of the movement-detector filter.

\section{ACKNOWLEDGMENTS}

We wish to thank K. G. Götz, W. Reichardt, and J. Zanker for critically reading the manuscript. Our thanks also are due to F. Buchstäber for drawing the figures and to U. Flaiz and I. Geiss for secretarial assistance.

\section{REFERENCES}

1. W. Reichardt, "Autocorrelation, a principle for evaluation of sensory information by the central nervous system," in Principles of Sensory Communication, W. A. Rosenblith, ed. (Wiley, New York, 1961), pp. 303-317.

2. W. Reichardt, "Evaluation of optical motion information by movement detectors," J. Comp. Physiol. A 161, 533-547. (1987).

3. E. Buchner, "Behavioral analysis of spatial vision in insects," in Photoreception and Vision in Invertebrates, M. A. Ali, ed. (Plenum, New York, 1984), pp. 561-621.

4. K. Nakayama, "Biological image motion processing: a review," Vision Res. 25, 625-660 (1985).

5. E. C. Hildreth and C. Koch, "The analysis of visual motion: from computation theory to neuronal mechanisms," Annu. Rev. Neurosci. 10, 477-533 (1987).

6. B. Hassenstein and W. Reichardt, "Systemtheoretische Analyse der Zeit-, Reihenfolgen- und Vorzeichenauswertung bei der Bewegungsperzeption des Rüsselkäfers Chlorophanus," Z. Naturforsch. 11b, 513-524 (1956).

7. W. Reichardt, "Autokorrelations-Auswertung als Funktionsprinzip des Zentralnervensystems (bei der optischen Wahrnehmung eines Insektes)," Z. Naturforsch. 12b, 448-457 (1957).

8. W. Reichardt and D. Varjú, "Übertragungseigenschaften im Auswertesystem für das Bewegungssehen (Folgerungen aus Experimenten an dem Rüsselkäfer Chlorophanus viridis)," Z. Naturforsch. 14b, 674-689 (1959).

9. D. Varjú, "Optomotorische Reaktionen auf die Bewegung periodischer Helligkeitsmuster (Anwendung der Systemtheorie auf Experimente am Rüsselkäfer Chlorophanus viridis)," Z. Naturforsch. 14b, 724-735 (1959).

10. A. J. van Doorn and J. J. Koenderink, "Temporal properties of the visual detectability of moving spatial white noise," Exp. Brain Res. 45, 179-188 (1982).

11. A. J. van Doorn and J. J. Koenderink, "Spatial properties of the visual detectability of moving white noise," Exp. Brain Res. 45, 189-195 (1982).

12. J. P. H. van Santen and G. Sperling, "Temporal covariance model of human motion perception," J. Opt. Soc. Am. A 1, 451473 (1984).

13. H. R. Wilson, "A model for direction selectivity in threshold motion perception," Biol. Cybern. 51, 213-222 (1985).

14. L. B. Baker and O. J. Braddick, "Temporal properties of the short-range process in apparent motion," Perception 14, 181192 (1985).

15. J. P. H. van Santen and G. Sperling, "Elaborated Reichardt detectors," J. Opt. Soc. Am. A 2, 300-321 (1985). 
16. E. H. Adelson and J. R. Bergen, "Spatiotemporal energy models for the perception of motion," J. Opt. Soc. Am. A 2, 284-299 (1985).

17. A. B. Watson and A. J. Ahumada, Jr., "Model of human visualmotion sensing," J. Opt. Soc. Am. A 2, 322-342 (1985).

18. J. Thorson, "Dynamics of motion perception in the desert locust," Science 145, 69-71 (1964).

19. W. Reichardt and A. Guo, "Elementary pattern discrimination (behavioural experiments with the fly Musca domestica)," Biol. Cybern. 53, 285-306 (1986).

20. M. Egelhaaf and W. Reichardt, "Dynamic response properties of movement detectors: theoretical analysis and electrophysiological investigation in the visual system of the fly," Biol. Cybern. 56, 69-87 (1987)

21. A. Borst and S. Bahde, "What kind of movement detector is triggering the landing response of the housefly?" Biol. Cybern. 55, 59-69 (1986).

22. W. Reichardt, "Processing of optical information by the visual system of the fly," Vision Res. 26, 113-126 (1986).

23. K. Hausen, "The lobula-complex of the fly: structure, function and significance in visual behaviour," in Photoreception and Vision in Invertebrats, M. A. Ali, ed. (Plenum, New York, 1984), pp. 523-559.

24. K. Hausen, "Motion sensitive interneurons in the optomotor system of the fly. I. The horizontal cells: structure and signals," Biol. Cybern. 45, 143-156 (1982).

25. N. Franceschini and K. Kirschfeld, "Les phénomènes de pseudopupille dans l'oeil composé de Drosophila," Kybernetik 9, 159-182 (1971).

26. A. Borst and S. Bahde, Max-Planck-Institut für Biologische Kybernetik, Spemanstrasse 38, D-7400 Tübingen, Federal Republic of Germany (personal communication).

27. K. Hausen, "Motion sensitive interneurons in the optomotor system of the fly. II. The horizontal cells: receptive field organization and response characteristics," Biol. Cybern. 46, 67-79 (1982).

28. R. R. de Ruyter van Steveninck, W. H. Zaagman, and H. A. K. Mastebroek, "Adaptation of transient responses of a movement-sensitive neuron in the visual system of the blowfly Calliphora erythrocephala," Biol. Cybern. 54, 223-236 (1986).

29. A. Borst and M. Egelhaaf, "Temporal modulation of luminance adapts time constant of fly movement detectors," Biol. Cybern. 56, 209-215 (1987).

30. K. Kirschfeld, "The visual system of Musca: studies on optics, structure and function," in Information Processing in the Visual System of Arthropods, R. Wehner, ed. (Springer-Verlag, Berlin, 1972), pp. 61-74.

31. K. G. Götz, "Optomotorische Untersuchung des visuellen Systems einiger Augenmutanten der Fruchtfliege Drosophila," Kybernetik 2, 77-92 (1964).

32. D. J. Tolhurst, "Separate channels for the analysis of the shape and the movement of a moving visual stimulus," J. Physiol. 231, 385-402 (1973).

33. A. Pantle, "Motion aftereffect magnitude as a measure of the spatio-temporal response properties of direction-sensitive analyzer," Vision Res. 14, 1229-1236 (1974).

34. H. C. Diener, E. R. Wist, J. Dichgans, and T. Brandt, "The spatial frequency effect on perceived velocity," Vision Res. 16, 169-176 (1976).

35. D. H. Kelly, "Motion and vision. II. Stabilized spatio-temporal threshold surface," J. Opt. Soc. Am. 69, 1340-1349 (1979).

36. D. C. Burr and J. Ross, "Contrast sensitivity at high velocities," Vision Res. 22, 479-484 (1982).

37. S. J. Anderson and D. C. Burr, "Spatial and temporal selectivity of the human motion detection system," Vision Res. 25, 11471154 (1985).

38. M. J. Wright and A. Johnston, "Invariant tuning of motion aftereffect," Vision Res. 25, 1947-1955 (1985).
39. T. Maddess and S. B. Laughlin, "Adaptation of the motionsensitive neuron $\mathrm{H} 1$ is generated locally and governed by contrast frequency," Proc. R. Soc. London Ser. B 225, 251-275 (1985).

40. H. Eckert and K. Hamdorf, "The contrast frequency-dependence: a criterion for judging the non-participation of neurones in the control of behavioural responses," J. Comp. Physiol. 145, 241-247 (1981).

41. T. Maddess, "Adaptive processes affecting the response of the motion sensitive neuron $\mathrm{H} 1$," in Proceedings of the International 1985 Conference on Cybernetics and Society (Institute of Electrical and Electronics Engineers, New York, 1985), pp. $862-866$.

42. T. Maddess, "Afterimage-like effects in the motion-sensitive neuron H1," Proc. R. Soc. London Ser. B 228, 433-459 (1986).

43. A. M. Derrington and G. B. Henning, "Errors in direction-ofmotion discrimination with complex stimuli," Vision Res. 27, 61-75 (1987).

44. C. Enroth-Cugell and J. G. Robson, "The contrast sensitivity of retinal ganglion cells of the cat," J. Physiol. 187,517-552 (1966).

45. S. B. Laughlin and R. C. Hardie, "Common strategies for light adaptation in the peripheral visual systems of fly and dragonfly," J. Comp. Physiol. 128, 319-340 (1978).

46. S. B. Laughlin, "Form and function in retinal processing," Trends Neurosci. 10, 478-483 (1987).

47. E. Buchner, "Elementary movement detectors in an insect visual system," Biol. Cybern. 24, 85-101 (1976).

48. B. P. M. Lenting, H. A. K. Mastebroek, and W. H. Zaagman, "Saturation in a wide-field, directionally selective movement detection system in fly vision," Vision Res. 24, 1342-1347 (1984).

49. H. Bülthoff and K. G. Götz, "Analogous motion illusion in man and fly," Nature 278, 636-638 (1979).

50. K. G. Götz, "Behavioral analysis of the visual system of the fruitfly Drosophila," in Proceedings of the Symposium on Information Processing in Sight Sensory Systems (California Institute of Technology, Pasadena, Calif., 1965), pp. 85-100.

51. A. Borst and S. Bahde, "Comparison between the movement detection systems underlying the optomotor and the landing response in the housefly," Biol. Cybern. 56, 217-224 (1987).

52. H. Wagner, "Aspects of the free flight behaviour of houseflies (Musca domestica)," in Insect Locomotion, M. Gewecke and G. Wendler, eds. (Paul Parey Verlag, Berlin, 1985), pp. 223-232.

53. M. Egelhaaf, "Dynamic properties of two control systems underlying visually guided turning in house-flies," J. Comp. Physiol. A 161, 777-783 (1987).

54. H. Wagner, "Flight performance and visual control of flight of the free-flying housefly (Musca domestica L.) III. Interactions between angular movement induced by wide- and smallfield stimuli," Philos. Trans. R. Soc. London Ser. B 312, 581-595 (1986).

55. C. Wehrhahn, T. Poggio, and H. Bülthoff, "Tracking and chasing in houseflies (Musca). An analysis of 3-D flight trajectories," Biol. Cybern. 45, 123-130 (1982).

56. J. O. Limb and J. A. Murphy, "Estimating the velocity of moving objects in television signals," Comput. Graphics Image Process. 4, 311-327 (1975).

57. S. Ullman, "Analysis of visual motion by biological and computer systems," Computer 14, 57-69 (1981).

58. V. Torre and T. Poggio, "A synaptic mechanism possibly underlying directional selectivity to motion," Proc. R. Soc. London Ser. B 202, 409-416 (1978).

59. N. Grzywacz and C. Koch, "Functional properties of models for direction selectivity in the retina," Synapse 1, 417-434 (1987).

60. A. Schmid and H. Bülthoff, "Using neuropharmacology to distinguish between excitatory and inhibitory movement detection mechanisms in the fly Calliphora erythrocephala," Biol. Cybern. 59, 71-80 (1988). 